\title{
Aerial Display Using Lenticular Lenses
}

\author{
Kazuhisa Yanaka, Kouki Shibata, Toshiaki Yamanouchi \\ Kanagawa Institute of Technology \\ 1030 Shimo-ogino, Atsugi-shi, Kanagawa-ken, Japan \\ yanaka@ic.kanagawa-it.ac.jp; s1723029@gmail.com; yama@ic.kanagawa-it.ac.jp
}

\begin{abstract}
An aerial display that can float a two-dimensional image or a three-dimensional object in an empty space is important as a noncontact interface. Aerial displays have two types: a reflection type that uses a mirror and a refraction type that involves a lens. The latter has the advantage of being easily miniaturized because the path of light does not bend much. The method using two commercially available lenticular lenses is inexpensive. However, the principle of the method is not yet fully understood. In this study, the rules of light rays passing through the lens were applied to explain this principle clearly. For further confirmation, a simulation was performed. Results revealed that the lens group consisting of two convex lenses arranged at the interval of twice the focal length as well as the lens group consisting of three convex lenses arranged at intervals the focal length reflected parallel rays coming from an angle as if they were mirrors. When the number of lenses was set to 3, the viewing range widened.
\end{abstract}

Keywords: aerial display, lenticular lens, Snell's law, refraction

\section{Introduction}

An aerial display that can float two-dimensional images and three-dimensional objects in air can attract the attention of customers when it is used for digital signage. It can also be used to operate electronic devices without contact, so it is also suitable for medical and cooking situations. To date, various aerial displays have been proposed.

Maekawa, Nitta and Matoba [1] proposed a method of making a large number of small square holes in a metal plate and using adjacent two of the four inner walls of each hole as a dihedral corner reflector. This special optical component is called a transmissive mirror device (TMD) or a parity mirror [2]. ASKA3D [3] also uses a dihedral corner reflector, but its structures and materials are different. In ASKA3D, the plate consists of two layers stacked orthogonally. Each layer consists of a number of small glass or plastic mirrors that are spaced at regular intervals similar to those of dominoes. The light that enters the plate is reflected once in each layer and goes out.

Yamamoto, Tomiyama and Suyama [4] proposed aerial imaging by retroreflection technology, which does not use dihedral corner reflectors. Instead, a retroreflector and a half mirror are used together. The light emitted from a point on an object is reflected by a retroreflecter and returns to its original position on the object. If a half mirror is inserted into the optical path at this time, a part of the returning light is reflected by the half mirror, and a real image is formed at a point different from the original point. Therefore, the real image can be seen in an empty space.

In this paper, the above methods are collectively called the reflection method because the mirror, not the lens, is used. The reflection method has an advantage of producing undistorted aerial images, since a lens has aberrations, but a mirror does not. On the other hand, aerial displays consisting of lenses rather than mirrors have already been proposed. In this paper, this kind of method is called the refraction method. The Floating Vision [5] commercialized by Pioneer seems to be an example of the refraction method because it uses special optics similar to a fly's eye lenses. There is another example. Kira and Yanaka [6] designed an aerial display by using double-sided lenticular lenses or a fly's eye lens sheets. Singlesided lenticular lenses are commercially available because demands for 3D printing are high, but double-sided lenticular lenses are not (Fig. 1). However, if two single-sided lenticular lenses are stacked so that flat surfaces are in contact with each other, they can be used as a double-sided lenticular lens. Unlike reflection methods, refractive methods have the advantage of not requiring a large space to bend the light because lenticular lenses should be placed on the LCD, and the paths of light rays are basically linear as shown in Fig. 2.

From this point onward, our discussion is narrowed down to the method involving lenticular lenses. 


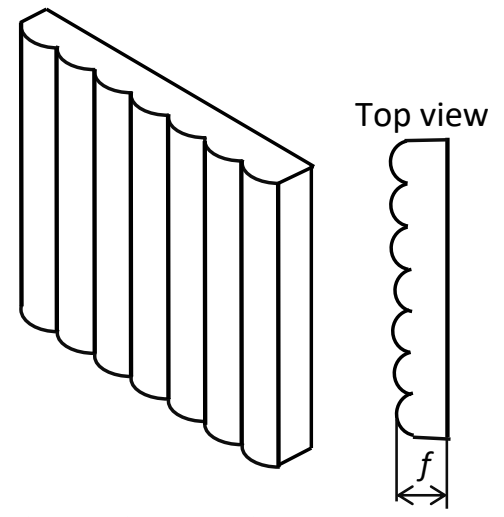

Single-sided lenticular lens

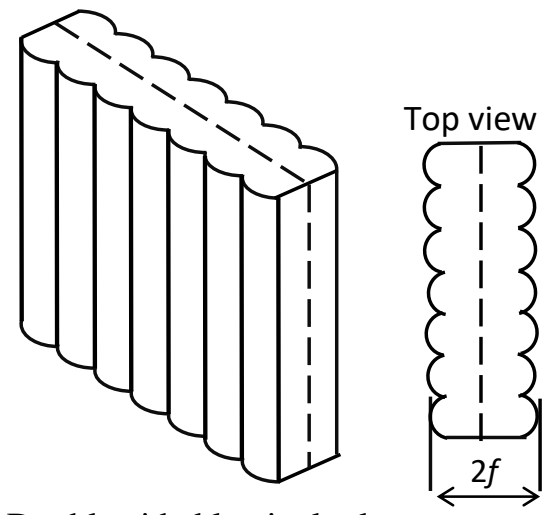

Double-sided lenticular lens

Fig. 1: Lenticular Lenses

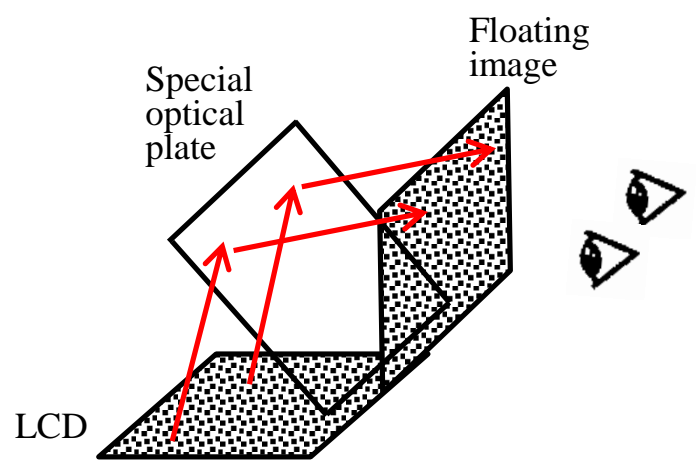

Reflection Method

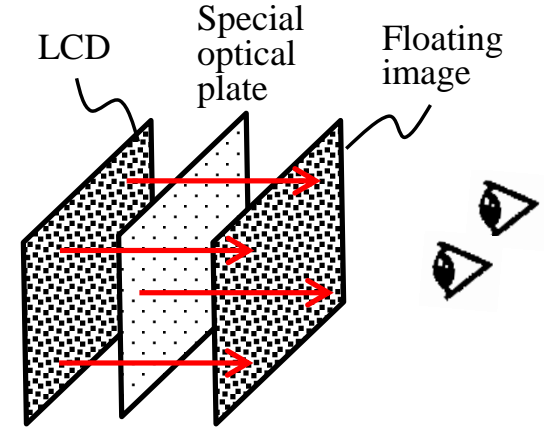

Refraction Method

Fig. 2: Reflection Method and Refraction Method

\section{Aerial display using two lenticular lenses}

Although the mechanism of the aerial display with double-sided lenticular lenses has been described in [6], not all experimental results have been explained yet. However, various laws have long been known about how light rays entering a convex lens are refracted, so we use them to explain the principle of this aerial display.

\subsection{Parallel Light Rays}

In Fig. 3, rays emitted from one point on an object can be regarded as almost parallel rays at the lens position far enough away. Therefore, we consider the direction of the parallel rays only.

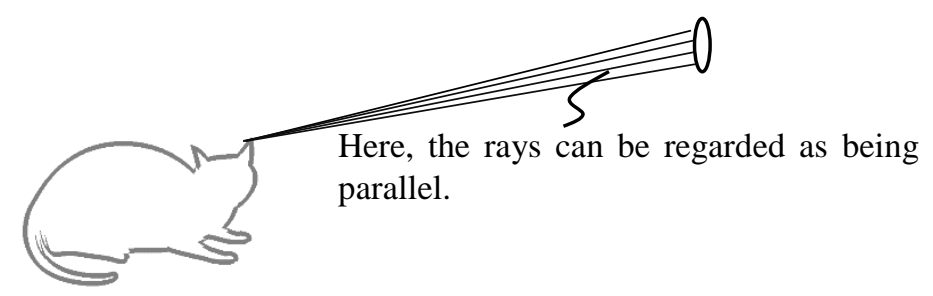

Fig. 3: Rays are almost parallel away from the light source. 


\subsection{Principle}

Fig. 4 shows the principle of the system involving two lenticular lenses. In Fig. 4 (a), two thin convex lenses of the same focal length are arranged on the same optical axis, and the distance between them is twice the focal length $f$ of the lens. Light emitted from a point on the object becomes parallel rays and enters the lens 1 at an angle $\alpha$. Among these parallel rays, we pay attention to a specific ray passing through the focal point $F_{1}$ on the front side of the lens 1 . This light ray enters the lens 1 at the point $P$, becomes parallel to the optical axis, passes through the point $\mathrm{Q}$, and enters lens 2 at the point R. For lens 2, this ray enters parallel to the optical axis, so it passes through the focal point $F_{2}$ after leaving the lens 2. Other emitted light rays drawn with dashed lines are parallel to this ray because they all come through point Q on the focal plane.

Triangles $\mathrm{F}_{1} \mathrm{PC}_{1}$ and $\mathrm{F}_{2} \mathrm{RC}_{2}$ are congruent, so $\alpha=\beta$. Thus, in Fig. 4 (b), this optical system reflects light rays like a mirror does.

Fig. 4 (c) shows an array of optical elements having a mirror function. Light emitted from any one point on the object collects at the position of the mirror image on the opposite side, resulting in an aerial display. However, binocular parallax cannot be obtained unless both eyes are at the same height. Otherwise the image does not float in air. If a large number of optical elements are arranged in a two-dimensional pattern, and if each element is changed to a dihedral corner reflector, Fig. 4 (c) becomes the same as a conventional aerial display.

Generally, commercially available lenticular lenses are not thin. They usually have thickness equal to the focal length. The configuration of the lens in that case is shown in Fig. 4 (d).

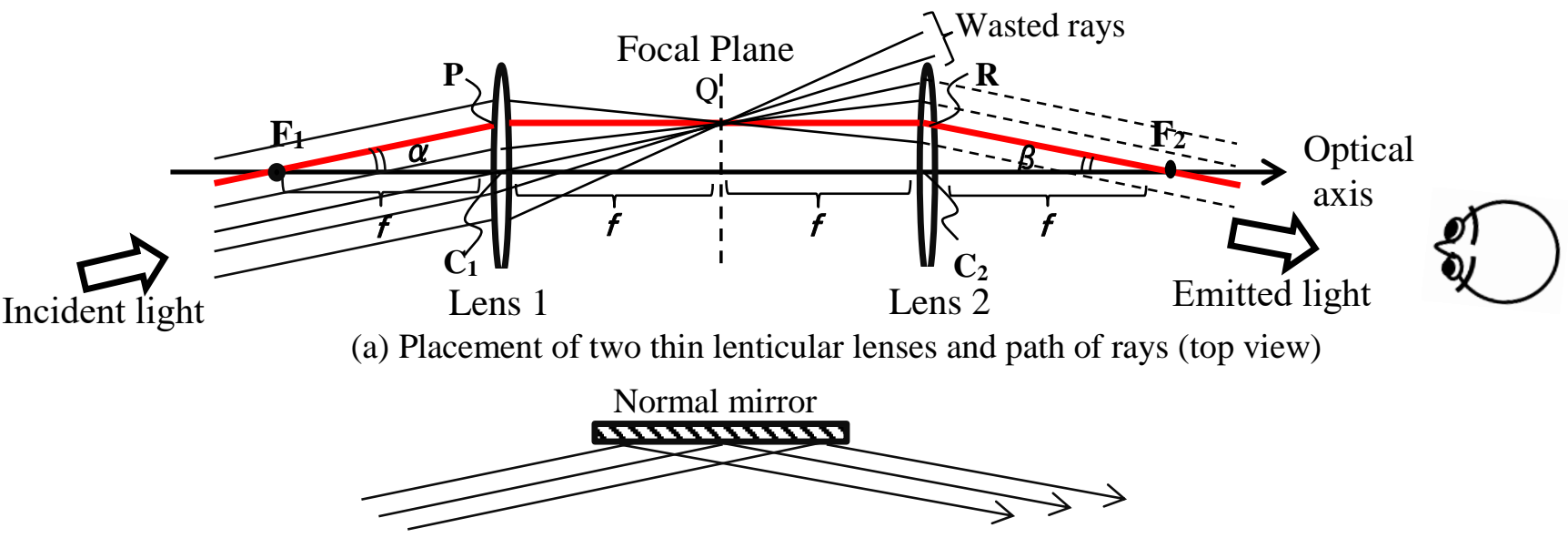

(b) Mirror optics almost equivalent to (a) (top view)
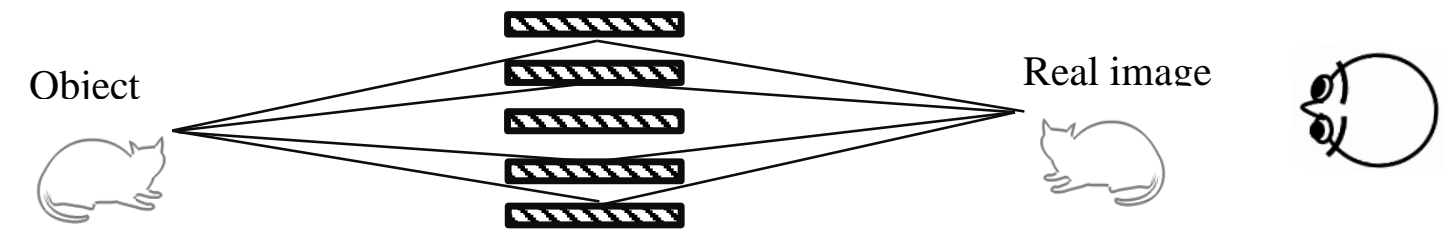

(c) Principle of generating a real image of a point light source by using a lens array or a mirror array (top view)

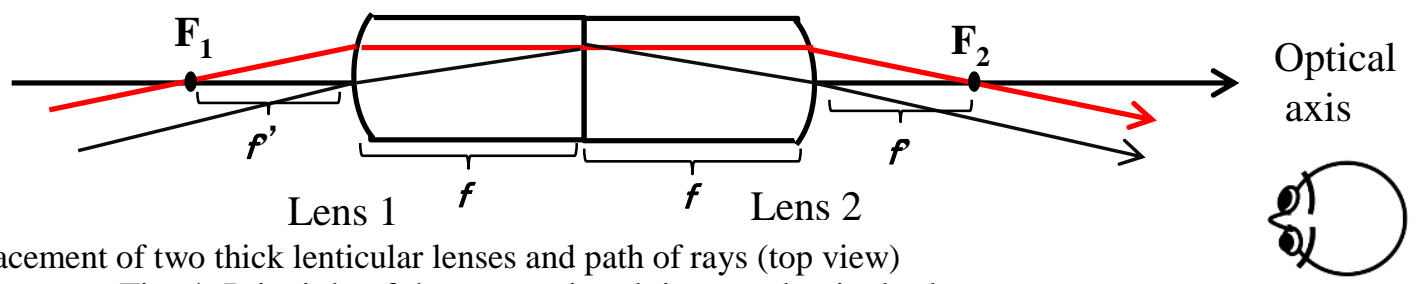

Fig. 4: Principle of the system involving two lenticular lenses 


\subsection{Simulation}

A simulation was conducted to find the path of light rays reflected at the surfaces of the lenses by applying Snell's law only. As a result, a real image was formed at the position of the mirror image with respect to the lenticular lenses (Fig. 5).

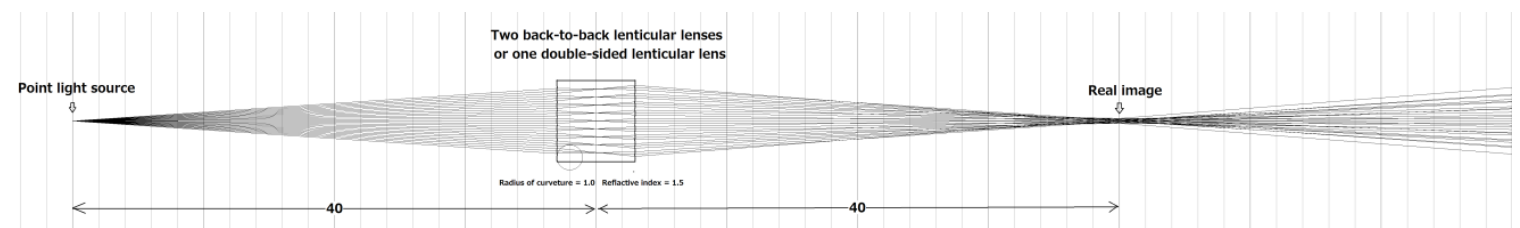

(a) Case of the distance between the point light source and the lenticular lens is 40. (top view)

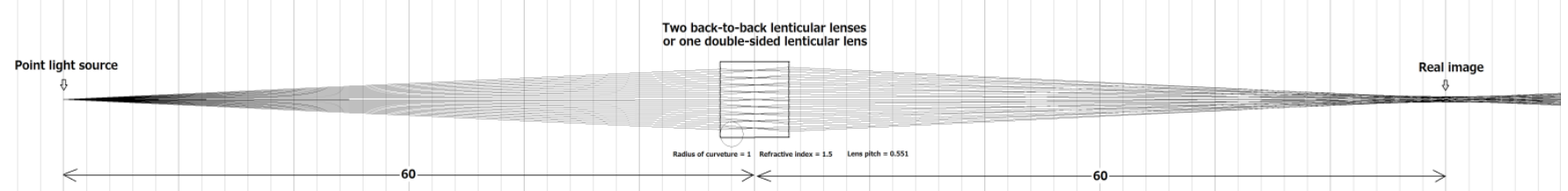

(b) Case of the distance between the point light source and the lenticular lens is 60. (top view)

Fig. 5: Simulation results

\subsection{Experiment}

In the method using two lenticular lenses, a clock placed behind the lenticular lenses appears to be popping out (Fig. 6).

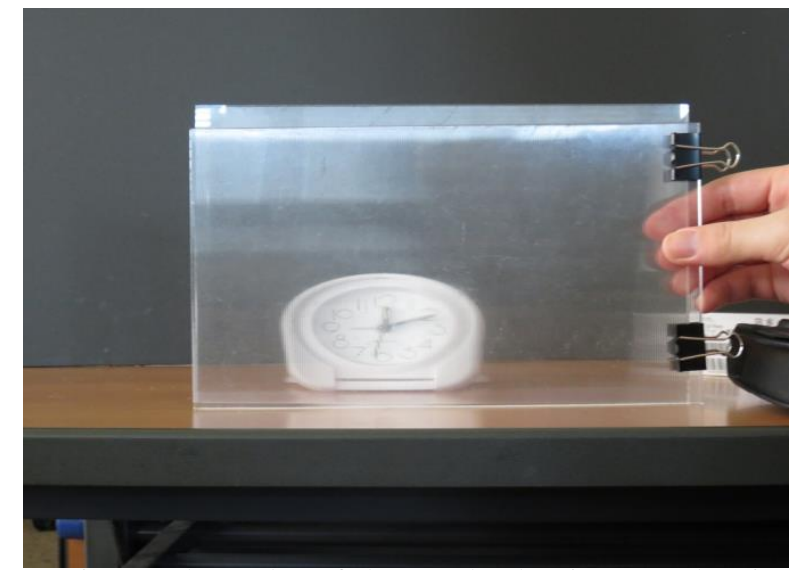

Fig. 6: Experimental results of the method using two lenticular lenses

\section{Aerial Display Using Three Lenticular Lenses}

In the method using two lenticular lenses, wasteful light is generated, as shown in Fig. 4 (a). Therefore, the viewing angle is narrowed. This problem can be improved by inserting Lens $M$ at the position of the focal plane in Fig. 4(a), as shown in Fig. 7 (a). The focal lengths of the three lenses are the same. Lens M refracts the light rays in the direction closer to the optical axis, so fewer light rays are wasted, and the viewing zone is widened. Incident rays are parallel to one another; as such, they all pass through point Q. All the light rays that have passed through point Q and entered lens 2 become parallel rays via lens 2 . Among them there is a light ray that passes through the focal point $F_{1}$ of lens 1. This ray enters lens 1 at point $\mathrm{P}$ and travels parallel to the optical axis. There is another ray that passes through the centre $C_{1}$ of the lens 1 . This ray is not refracted at $C_{1}$ but go straight. The ray is refracted by Lens $M$ at point $Q$ and becomes parallel to the optical axis. It is because point $\mathrm{C}_{1}$ is also a focal point of Lens $\mathrm{M}$. The lengths of straight lines 
$\mathrm{PC}_{1}$ and $\mathrm{RC}_{2}$ are equal because straight lines $\mathrm{PQ}$ and $\mathrm{QR}$ are both parallel to the optical axis. Therefore, triangles $\mathrm{F}_{1} \mathrm{PC} \mathrm{C}_{1}$ and $\mathrm{F}_{2} \mathrm{RC}_{2}$ are congruent, and $\alpha=\beta$. This result indicates that this optical system also reflects light rays like a mirror does, as shown in Fig. 4 (b). From this point onward, the discussion is basically the same as described in the previous chapter.

Commercially available lenticular lenses are not thin. They usually have thickness equal to the focal length. The configuration of the lens in that case is shown in Fig. 7 (b).

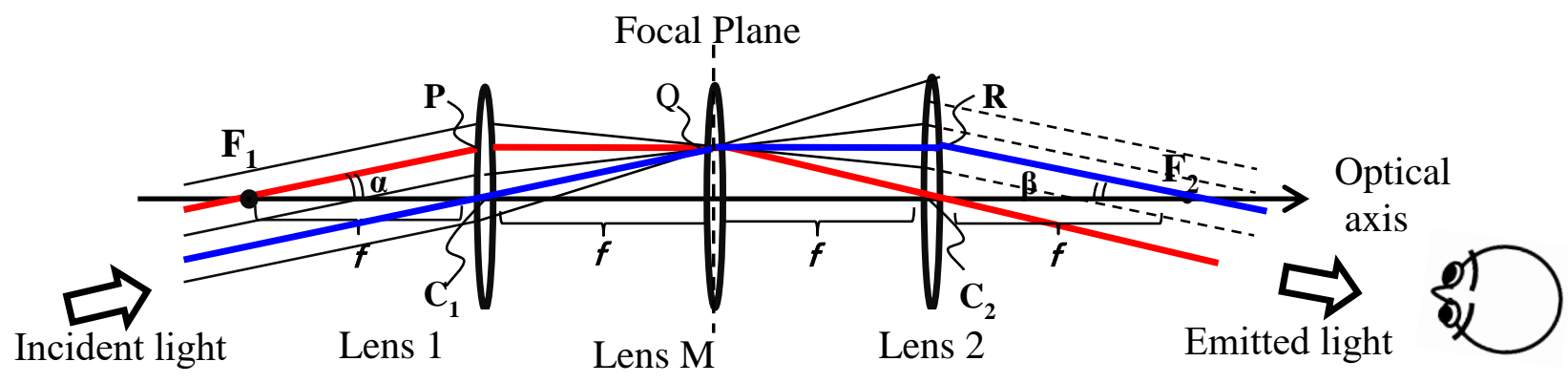

(a) Placement of three thin lenticular lenses and path of rays (top view)

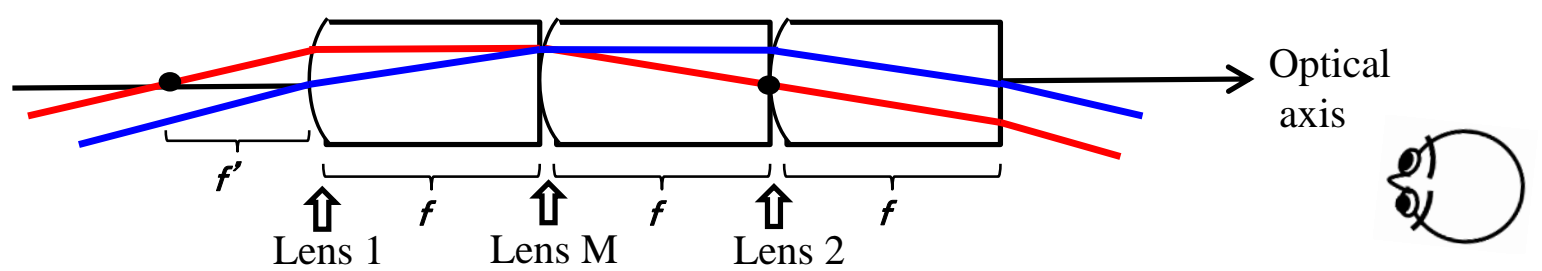

(b) Placement of three lenticular lenses and path of rays

Fig. 7: Principle of the system involving three lenticular lenses

The simulation results are shown in Fig. 8. Similar to the method using two lenticular lenses, this method forms a real image at the position of the mirror image. The experimental results are presented in Fig. 9. This method has an increased angle for stereoscopic viewing compared with the method involving two lenticular lenses, which allow stereoscopic viewing only from the front.

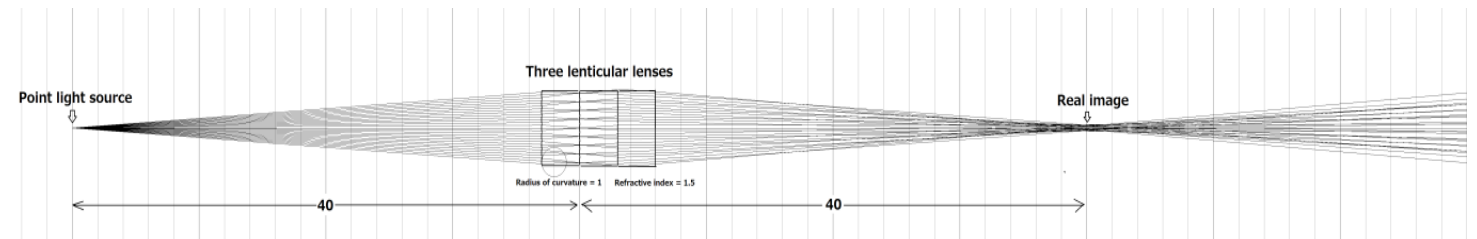

(a) Case of the distance between the point light source and the lenticular lens is 40. (top view)

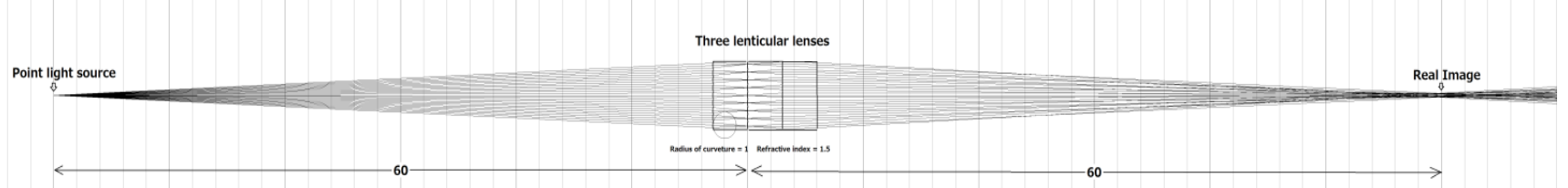

(b) Case of the distance between the point light source and the lenticular lens is 60. (top view)

Fig. 8: Simulation results 


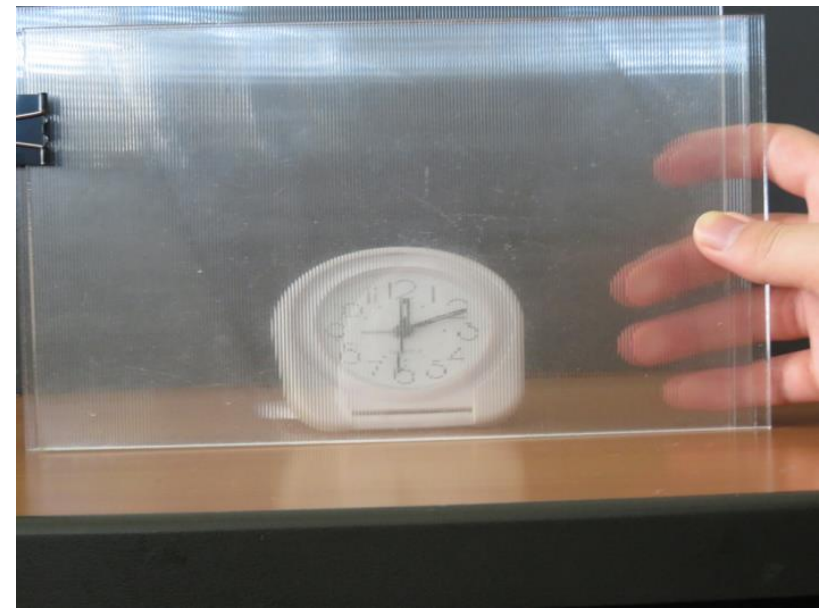

Fig. 9: Experimental results with three lenticular lenses

\section{Conclusion}

As for an aerial display using two lenticular lenses or one double-sided lenticular lens, the principle was clarified by applying the rules for the light rays entering the lenses. We found that two lenses whose interval was 2 times the focal length and three lenses whose interval was the same as the focal length had the same function as reflecting incident parallel light like a mirror. Therefore refraction and reflection aerial displays had unexpected similarities. Simulations were also performed to confirm the theoretically elucidated principles. A new method was proposed to increase the field of view by increasing the number of lenticular lenses from two to three. It was a lenticular system, so it had limitations. For instance, a screen would not pop out when the head was tilted 90 degrees. If the lenticular lens was changed to a fly's eye lens, this problem would disappear. This system is promising because lenticular lenses are cheaply available commercially, and the installation space can be saved because there is no need for a large space to bend the rays.

\section{References}

[1] Satoshi Maekawa, Kouichi Nitta, Osamu Matoba, "Transmissive optical imaging device with micromirror array," in proceedings of Optics East 2006.

[2] Parity Innovations, [Online]. Available: https://www.piq.co.jp/index_e.html.

[3] ASKA3D [Online]. Available: https://aska3d.com/en/technology.html

[4] Hirotsugu Yamamoto, Yuka Tomiyama, and Shiro Suyama, "Floating aerial LED signage based on aerial imaging by retro-reflection (AIRR)," Optics Express, vol. 22, issue 22, pp. 26919-26924 (2014). Also available: https://www.osapublishing.org/oe/fulltext.cfm?uri=oe-22-22-26919\&id=303352

[5] Masaru Ishikawa, "A proposal to create the impression of a miniature garden, and the development of a glasses-free small 3-D display," Pioneer R\&D vol.12, no.3, pp. 47-58, 2003. (in Japanese)

[6] Naoki Kira and Kazuhisa Yanaka, "Spatial Image Display using Double-sided Lenticular or Fly's Eye Lens Sheets," in Proceedings of VISAPP2013, pp.425-428, 2013. 